\title{
Konsensuspapier zur Aknetherapie Topische Retinoide als Behandlung erster Wahl
}

D ie Therapie der Akne verläuft oft unbefriedigend. Dies gilt nicht nur für den einzelnen Patienten, sondern auch weltweit gesehen. Abzulesen ist dies beispielsweise an der zunehmenden Resistenzentwicklung von P. acnes. Um einen einheitlichen evidenzbasierten Behandlungsstandard zu ermöglichen, wurde im Sommer 2003 ein Konsensus-Papier der „Global Alliance to Improve Outcomes in Acne" veröffentlicht. Einige der beteiligten Experten stellten die Ergebnisse auf dem EADV in Barcelona vor.

Das Konsensuspapier wurde erstellt, um weltweit das Verschreibungsverhalten der Ärzte zu verändern, so Prof. Harald Gollnick aus Magdeburg. Nur so könne die zunehmende Resistenz der Aknebakterien gestoppt und eindeutige Daten international akzeptiert werden. Die Kernaussage des Papiers: Die Therapie der Komedonen-Akne und der leichten bis mittelschweren entzündlichen Akne sollte mit topischen Retinoiden - allein oder in Kombination - durchgeführt werden. Hiervon profitieren die meisten (ca. 85\%) der Patienten, so Prof. James Leyden aus Philadelphia. Die Therapie mit einem topischen Retinoid wie Adapalen ${ }^{1}$ sollte als erster Therapieschritt und möglichst schnell erfolgen, auch um eine Narbenbildung zu verhindern. Bei mittelschwerer Akne wirkt die Kombination von topischen Retinoiden mit Antibiotika schneller und signifikant besser als eine antibiotische Monotherapie. Hierzu wurden mehrere Studien veröffentlicht, so Leyden. Durch die Kombination kann daher die Dauer des Antibiotikaeinsatzes verkürzt werden. Für die schweren Aknefälle sieht der Konsensus dagegen Isotretinoin vor.

\section{Erhaltungstherapie wichtig}

Topische Retinoide wirken dabei auf mehreren Ebenen gegen Akne.

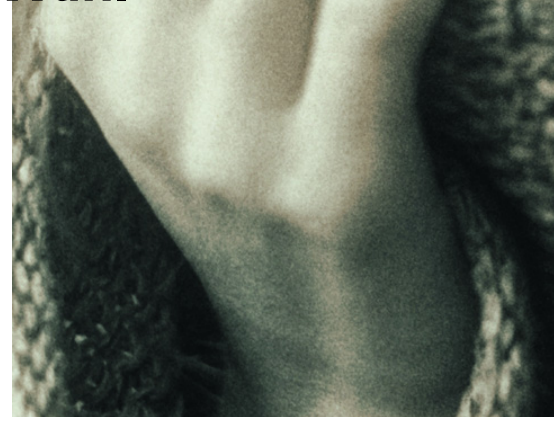

Sie hemmen die Neubildung von Mikrokomedonen und vermindern die Anzahl bestehender Komedonen. Weiterhin fördern sie die normale Desqamation des follikulären Epithels. Erst seit einigen Jahren weiß man auch, dass die Retinoide selber eine nicht unerhebliche antimikrobielle Wirkung zeigen, betonte Leyden. Dies geht bei den modernen Präparaten wie Adapalen mit einer guten Verträglichkeit einher, der Voraussetzung für einen längerfristigen Einsatz.

Wichtig für die Anwendung, so Gollnick, ist es, die Retinoide auf das ganze Areal aufzutragen und nicht nur auf einzelne Läsionen. Auch nach einer Besserung sollte eine Erhaltungstherapie fortgeführt werden. Diese kann flexibel gestaltet werden. Laut Gollnick muss getestet werden, ob das Retinoid täglich, jeden zweiten Tag oder zweimal die Woche aufgetragen werden sollte. Um eine übersichtliche Therapiempfehlung geben zu können, wurde von der Konsensusgruppe ein Algorithmus entwickelt, der weltweit erhältlich ist. Dieser zeigt für verschiedene Erkrankungsstadien jeweils die erste Therapiewahl sowie Alternativen auf.

\section{Lebensqualität wird stark verbessert}

Gollnick betonte, dass auch die Lebensqualität der Patienten durch eine effektive Behandlung dramatisch verbessert werden könne. Wichtig sei im Vorfeld, sich viel Zeit für den Patienten zu nehmen und ihn ausreichend aufzuklären, da eine gute Compliance für die Therapie unbedingt notwendig ist. Prof. William Cunliffe aus Leeds belegte die große Bedeutung mit Zahlen: die effektive Mitarbeit der Patienten macht bei einigen Therapieoptionen bis zu $40 \%$ des Erfolgs aus. Man sollte Aknebetroffenen unbedingt klarmachen, dass man ihre Erkrankung gut behandeln kann, dass sie aber nicht innerhalb einiger Tage verschwunden sei (wovon einige Patienten ausgehen und dann die Therapie frustriert abbrechen), sondern die Therapie oft jahrelang durchgeführt werden müsse. Der Patient soll möglichst von Anfang an in die Therapieentscheidung einbezogen werden. Dies geschehe derzeit in Großbritannien, so Cunliffe.

Einen Eindruck vom Gemütszustand gab eine von Cunliffe vorgestellte Fragebogenaktion aus Großbritannien, die eine relativ hohe Rücklaufquote von $48 \%$ hatte. Die Ergebnisse zeigen, dass sich die Patienten psychologisch stark gestresst und in ihrem täglichen Leben eingeschränkt fühlen, vor allem betroffen war natürlich der zwischenmenschliche Bereich. Deutlich zum Ausdruck kam die Verzweiflung bei der Frage, ob die Patienten 5000 englische Pfund oder eine komplette Heilung bevorzugen würden: $94 \%$ entschieden sich für die Heilung.

Sibylle Rettenmaier, Stuttgart

Quelle: Pressekonferenz „Changing the face of acne treatment", Barcelona, Oktober 2003, unterstützt von Galderma $\mathrm{GmbH}$, Freiburg.

1 Differin ${ }^{\circledR}$, Galderma GmbH 